\title{
\begin{tabular}{l|l} 
Mibraries & DSpace@MIT
\end{tabular}
}

\author{
MIT Open Access Articles
}

\section{VIV Excitation Competition Between Bare and Buoyant Segments of Flexible Cylinders}

The MIT Faculty has made this article openly available. Please share how this access benefits you. Your story matters.

Citation: Rao, Zhibiao, J. Kim Vandiver, and Vikas Jhingran. "VIV Excitation Competition Between Bare and Buoyant Segments of Flexible Cylinders." Volume 7: CFD and VIV (June 9, 2013).

As Published: http://dx.doi.org/10.1115/OMAE2013-11296

Publisher: American Society of Mechanical Engineers

Persistent URL: http://hdl.handle.net/1721.1/109278

Version: Final published version: final published article, as it appeared in a journal, conference proceedings, or other formally published context

Terms of Use: Article is made available in accordance with the publisher's policy and may be subject to US copyright law. Please refer to the publisher's site for terms of use. 


\title{
VIV EXCITATION COMPETITION BETWEEN BARE AND BUOYANT SEGMENTS OF FLEXIBLE CYLINDERS
}

\author{
Zhibiao Rao' \\ Department of Mechanical Engineering \\ Massachusetts Institute of Technology \\ Cambridge, MA, USA
}

\author{
Prof. J. Kim Vandiver \\ Department of Mechanical Engineering \\ Massachusetts Institute of Technology \\ Cambridge, MA, USA
}

\author{
Dr. Vikas Jhingran \\ Shell Exploration and Production International, \\ Houston, Texas, USA
}

${ }^{1}$ Corresponding Author, Zhibiao Rao, Email: zbrao@mit.edu

\begin{abstract}
This paper addresses a practical problem: "Under which coverage of buoyancy modules, would the Vortex Induced Vibration (VIV) excitation on buoyant segments dominate the response?" This paper explores the excitation competition between bare and buoyant segments of a 38 meter long model riser. The source of data is a recent model test, conducted by SHELL Exploration and Production at the MARINTEK Ocean Basin in Trondheim Norway. A pipe model with five buoyancy configurations was tested.
\end{abstract}

The results of these tests show that (1) the excitation on the bare and buoyant regions could be identified by frequency, because the bare and buoyant regions are associated with two different frequencies due to the different diameters; (2) a new phenomenon was observed; A third frequency in the spectrum is found not to be a multiple of the frequency associated with either bare or buoyancy regions, but the sum of the frequency associated with bare region and twice of the frequency associated with buoyancy region; (3) the contribution of the response at this third frequency to the total amplitude is small; (4) the power dissipated by damping at each excitation frequency is the metric used to determine the winner of excitation competition. For most buoyancy configurations, the excitation on buoyancy regions dominates the VIV response; (5) a formula is proposed to predict the winner of the excitation competition between bare and buoyant segments for a given buoyancy coverage.

\section{INTRODUCTION}

It is expected that the existence of buoyancy modules may decrease the fatigue damage rate due to the decrease in vortex shedding frequency for its larger diameter. A bare cylinder will vibrate at a higher frequency than a cylinder fully covered by buoyancy modules of a much larger diameter. When a flexible cylinder with both bare and buoyant regions is excited by the same flow, two different frequencies are excited and a competition exists between lift forces at these two frequencies. The point of this study was to answer the question, "Under which coverage of buoyancy, would the VIV excitation on buoyant regions dominate the response?"

There exists some previous research on the excitation competition between bare and buoyant segments of a pipe. Lie et al [1] used the RMS amplitude ratio associated with the buoyant segments divided by the total RMS to determine whether the excitation on buoyant regions dominates the VIV response. While Vandiver and Peoples [2], Li et al [3] and Vikas et al [4] used the frequencies of the peak spectral components to determine whether the excitation on buoyant regions dominates the VIV response. Their preliminary data analysis showed that the coverage of buoyancy modules and the ratio of two diameters play a major role in the excitation competition between bare and buoyant segments.

Lie et al [1] stated that the ratio between the lift force on the bare segments and that on the buoyant segments was proportional to the ratio of $\frac{L_{\text {bare }}}{L_{\text {buoyancy }}} \frac{D_{\text {bare }}}{D_{\text {buoyancy }}}$. Where $L_{\text {bare }}$ and $L_{\text {buoyancy }}$ are the length of covered bare and buoyant segments, 
respectively; $D_{\text {bare }}$ and $D_{\text {buoyancy }}$ are the outer diameter of bare and buoyancy segments, respectively. Vandiver [5] also came up with a similar formula, where the amplitude ratio between the modal responses due to the excitation on bare segments and buoyant segments was proportional to the ratio of $\left(\frac{L_{\text {bare }}}{L_{\text {buoyancy }}} \frac{D_{\text {bare }}}{D_{\text {buoyancy }}}\right)^{2}$. Both expressions show that when a pipe has two different diameters, the larger diameter region is favored to dominate the response.

This paper explores the excitation competition between bare and buoyant regions of a 38 meter long model riser. The power dissipated by the damping at each excitation frequency is the metric used to determine the winner. The distinguishing identifiable feature of the excitation on either region is the excitation frequency. Two excitation frequencies are well known to exist in the spectrum of the pipe with staggered buoyancy modules. The higher frequency is associated with bare regions and the lower one associated with buoyant regions according to the Strouhal relationship $f=S_{t} U / D$. In addition to these two excitation frequencies, a new phenomenon was observed. A third frequency was found to be an unexpected combination of these two excitation frequencies. This is the first known report of such behavior.

The main objectives of this paper are to:

(1) Explain the existence of the third frequency.

(2) Study the effect of the excitation at the third frequency on the VIV response.

(3) Determine the winner of the excitation competition between bare and buoyant regions.

(4) Propose an expression to predict the winner of the excitation competition for a pipe with staggered buoyancy modules under uniform flow.

\section{EXPERIMENT DESCRIPTION}

Riser and Buoyancy Configurations. The total pipe length was 38 meters. Pipe 2 had a diameter of $30 \mathrm{~mm}$ and was made from a continuous length of fiberglass tubing. Pipe 3 used pipe 2 as the inner core. Ninety-three pairs of clamshell modules, 80 $\mathrm{mm}$ in diameter, were clamped onto the outside of the $30 \mathrm{~mm}$ inner pipe. Each module was $0.4086 \mathrm{~m}$ in length and was made from two flexible urethane half shells which snapped together around the smaller Pipe 2 and secured with locking clips. Hereafter, Pipe 2 will also be called the medium pipe and the Pipe 3 will be referred as the large pipe. Different buoyancy configurations could be modeled by clamping on the modules, leaving bare gaps between module segments. The details are listed in Table 1.

Table 1: Pipe Model Properties

\begin{tabular}{|c|c|c|}
\hline Parameter & Pipe2 & Pipe3 \\
\hline Total length between pinned ends $(\mathrm{m})$ & 38.00 & 38.00 \\
\hline Outer diameter $(\mathrm{mm})$ & 30 & 80 \\
\hline
\end{tabular}

\begin{tabular}{|c|c|c|}
\hline $\begin{array}{c}\text { Outer/Inner diameter of fiberglass } \\
\text { rod/pipe }(\mathrm{mm})\end{array}$ & $27 / 21$ & $27 / 21$ \\
\hline The length of 1 buoyancy section $(\mathrm{m})$ & NA & 0.4086 \\
\hline Bending stiffness of Pipe $2, \mathrm{EI}\left(\mathrm{Nm}^{2}\right)$ & 572.3 & 572.3 \\
\hline Young modulus for Pipe $2, \mathrm{E}\left(\mathrm{N} / \mathrm{m}^{2}\right)$ & $3.46 \times 10^{10}$ & $3.46 \times 10^{10}$ \\
\hline Mass in air $(\mathrm{kg} / \mathrm{m})$ & 1.088 & 5.708 \\
\hline Weight in water $(\mathrm{kg} / \mathrm{m})$ & 0.579 & 0.937 \\
\hline Mass ratio & 1.54 & 1.14 \\
\hline
\end{tabular}

The bending stiffness of the urethane shells was considered negligible, compared to the effect of tension on the natural frequencies of the configurations tested. For the staggered buoyancy test, the length of an individual buoyant section was defined as $L_{b}$ and the gap between two adjacent segments was defined $L_{c}$. Figure 1 shows the five configurations of flexible cylinders with staggered buoyancy. In all tests the ratio between buoyancy element diameter and bare riser diameter was 2.67. This non-integer ratio prevents the bare riser vortex frequency from being an integer multiple of the vortex frequency from the buoyancy elements. In order to study the effect of the staggered buoyancy modules on VIV, the configuration matrix was populated with five different length ratios, namely $L_{c} / L_{b}=2 / 2, L_{c} / L_{b}=1 / 1, L_{c} / L_{b}=3 / 2, L_{c} / L_{b}=3 / 1$ and $L_{c} / L_{b}=5 / 2$. For example when the length is specified as $L_{c} / L_{b}$ $=2 / 2$, it means that $L_{b}=L_{c}=2 \times 0.4086$. This identification system has the advantage of identifying the both the length of the buoyant and bare segments, as well as the ratio of length of the buoyant segment to the bare or gap length5.

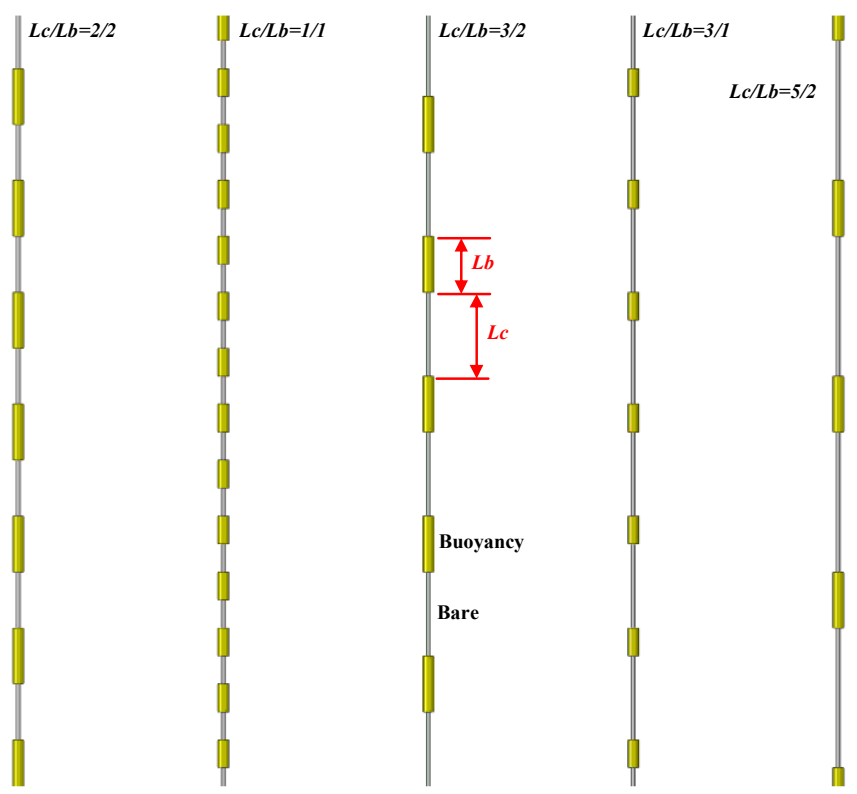

Figure 1. Section configurations of flexible cylinders with staggered buoyancy

Test Arrangement. The source of data is a recent model test, conducted by SHELL Exploration and Production at the 
MARINTEK Ocean Basin in Trondheim Norway (see [4] for more details). The test matrix consisted of 45 runs at varying towing velocities and varying configurations of buoyancy elements. Each buoyant configuration consisted of 9 runs at varying towing velocities. The pipe was exposed to uniform flow only. The maximum flow speed was up to $1.2 \mathrm{~m} / \mathrm{s}$ corresponding to a Reynolds number of $\sim 96000$ for $D_{\text {buoyancy }}=80 \mathrm{~mm}$. The pipe tension was approximately constant for each test in the steady state but varied with flow speed and the configuration from test to test. Mean tensions ranged from $5900 \mathrm{~N}$ to $7800 \mathrm{~N}$. The dominant excited mode for $D_{\text {bare }}=30 \mathrm{~mm}$ varied from 3 to 13 while the dominant excited mode for $D_{\text {buoyancy }}=80 \mathrm{~mm}$ varied from 1 to 5 .

The pipe was densely instrumented with 30 equally spaced strain gauges and 20 accelerometers in both cross flow (CF) and in line (IL) directions. In addition, two tri-axial force transducers were installed at both ends. All transducers were sampled at a rate of $1200 \mathrm{~Hz}$.

\section{ANALYSIS TECHNIQUES}

\section{Nonlinear Interaction between Two Excitation Frequencies}

If the system is linear, the frequency components in response will be the same as those in excitation forces. If the system is nonlinear, excitation frequency components will include those in the excitation forces plus additional frequencies. These additional frequencies are due to the nonlinear interaction of the response of the cylinder at the excitation frequency components. Hereafter, the additional frequency component is called the nonlinear interaction frequency.

For a flexible cylinder with staggered buoyancy modules, there are two main excitation frequencies; the higher frequency is associated with the excitation on bare regions and the lower one is associated with the excitation on buoyant regions. VIV is known to be a nonlinear phenomenon; it is reasonable to suspect that a nonlinear interaction frequency may exist.

A pipe with nonlinear damping and two excitation sources at different frequencies was analyzed in order to explore the additional frequency components presented in the response. The nonlinear interaction frequencies arising are due to the nonlinear terms in the governing equation. The mechanism for nonlinear interaction between two wave-trains has been extensively studied in nonlinear optics [6], plasma physics [7], fluid mechanics $[8,9]$, etc. In the next few sections a similar analysis is conducted for this hydrodynamic application using an asymptotic expansion technique.

\section{The equation of motion of a flexible riser}

It is believed that the nonlinear interaction frequencies are the result of the nonlinear damping term in the equation of motion. Here, a uniform distributed pipe with nonlinear damping is used as a model to study the effect of the nonlinear damping term on the response frequency components. The following analysis presents the key results. The equation of motion may be written as:

$$
m \frac{d^{2} y(x, t)}{d t^{2}}+c(x, t) \frac{d y(x, t)}{d t}+E I \frac{d^{4} y(x, t)}{d x^{4}}-T \frac{d^{2} y(x, t)}{d x^{2}}=F(x, t)(1)
$$

Where $m, E I$, and $T$ are the constant mass per unit length, bending stiffness and tension, respectively; $y(x, t)$ is the displacement in the cross-flow direction; $c(x, t)$ is the nonlinear damping per unit length; $F(x, t)$ is the excitation force.

In Equation 1, the hydrodynamic contribution to the damping term, $c(x, t)$, is assumed to come only from the power-out region. The lift force per unit length $F(x, t)$ is assumed to act only in the power-in region. Within power-in regions where the modal amplitude may be quite large, the lift coefficient may become negative, thus extracting power from the vibration. This is accounted for in the solution of equation 1 in the solution for the modal lift force. The damping term $c(x, t)$ accounts for hydrodynamic damping outside of the power-in region.

\section{Venugopal's hydrodynamic damping model}

The Venugopal hydrodynamic damping model ${ }^{[10]}$ was used to estimate the hydrodynamic damping in the power-out region. This model is also used to estimate the average power dissipated by VIV for the pipe with buoyancy modules in a later section of the paper.

(1) Hydrodynamic damping model when reduced velocity Vr $<7$

$$
\begin{gathered}
c=R_{s w}+C_{r l} \rho D U \\
R_{s w}=\frac{\omega \pi \rho D^{2}}{2}\left[\frac{2 \sqrt{2}}{\sqrt{R e_{\omega}}}+C_{s w}\left(\frac{A}{D}\right)^{2}\right] \\
=\frac{\omega \pi \rho D^{2}}{2}\left[\frac{2 \sqrt{2}}{\sqrt{R e_{\omega}}}+C_{s w}\left(\frac{\sqrt{2} Y(x)}{D}\right)^{2}\right]
\end{gathered}
$$

(2) Hydrodynamic damping model when reduced velocity $\boldsymbol{V r}>7$

$$
c=C_{r h} \rho U^{2} / \omega
$$

Where $R_{S w}$ is the still water contribution; $C_{r l}$ is an empirical coefficient taken to be $0.18 ; R e_{\omega}=\frac{\omega D^{2}}{v}$, a vibration Reynolds number; $C_{s w}$ and $C_{r h}$ are two other empirical coefficients, which at present are taken to be $0.2^{[10]}$.

In general, the hydrodynamic damping can be rewritten as:

$$
c(x, t)=c_{1}+c_{2} y^{2}(x, t)
$$


The damping in Eq.(5) is nonlinear because the response $y(x, t)$ influences the damping $c(x, t)$. The larger the response is, the greater the damping.

\section{Asymptotic expansion}

Suppose there are two main excitation frequencies, $\omega_{1}$ and $\omega_{2}$ in the excitation force. If the structural system is linear, the frequency components in the steady state response $y(x, t)$ will only contain $\omega_{1}$ and $\omega_{2}$. However because the structural system is nonlinear due to the existence of the nonlinear damping term, the following frequency components could be presented in the response: $3 \omega_{1}, 3 \omega_{2}, 2 \omega_{1}+\omega_{2}, 2 \omega_{1}-\omega_{2}$, $2 \omega_{2}+\omega_{1}$ and $2 \omega_{2}-\omega_{1}$. The detailed derivation for the possible additional frequency components can be found in the Appendix. It is not a necessary condition that all of these components appear in the response. Some may and some may not. When an additional frequency component appears in the response, this frequency and its corresponding wave number must satisfy the dispersion relation of the pipe.

\section{PRESENTATION OF DATA AND RESULTS}

\section{Frequency components in uniform bare pipe}

Excitation frequency is determined from the dominant peaks in the curvature power spectrum. Dating back to the 1980's, Vandiver and Chung ${ }^{[11]}$ reported that vibrations associated with the normally expected VIV excitation frequency were often accompanied by vibrations at integer multiples of this frequency. Of these, the odd multiples of three and five were often associated with the cross-flow direction.

Figure 2 shows the curvature spectrum of bare medium pipe (red line) and bare large pipe (blue dash). The data was the averaged spectrum of 30 different sensors at $1.0 \mathrm{~m} / \mathrm{s}$ uniform flow, corresponding to Test 3007 and Test 4004, respectively. In Figure 2 , several frequencies were observed.

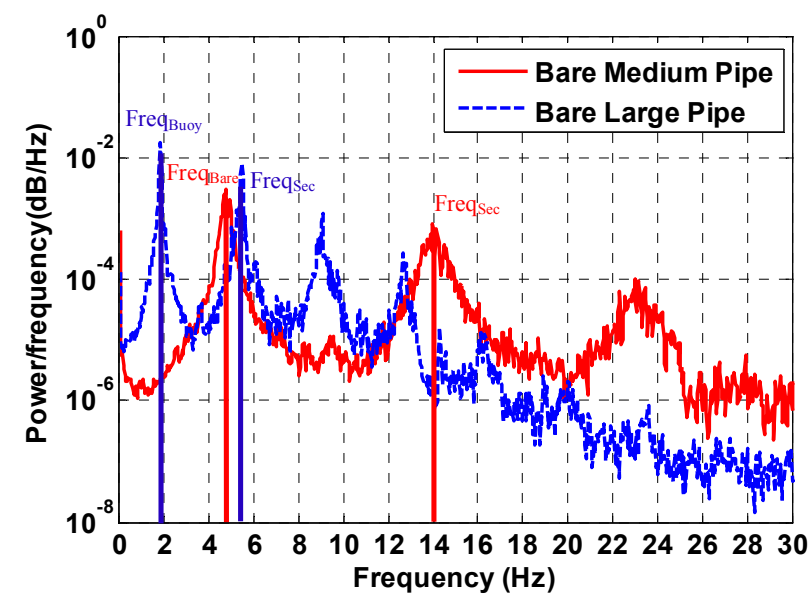

Figure 2: An example curvature power spectrum for bare medium pipe (red line) and bare large pipe (blue dash). The data was the averaged spectrum of 30 curvatures at $1.0 \mathrm{~m} / \mathrm{s}$ uniform flow, corresponding to Test 3007 and Test 4004, respectively.

By picking the highest and secondary peaks in the curvature power spectrum, a plot of these two frequencies versus flow speed for both bare medium and large pipes are shown in Figures 3 and 4 . The highest peak frequency is usually referred to the excitation frequency. In order to differentiate the excitation frequency for the bare medium and large pipes, "Freq Bare $_{\text {" and }}$

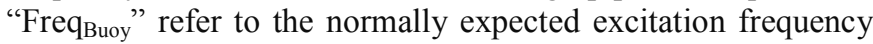
for the medium pipe and the large pipe, respectively. And the secondary peak frequency is expressed as "Freqsec". In Figures 3 and 4 , the green line represents the theoretical value of three times the normal excitation frequency for the pipe in the test. The black triangle represents the secondary peak frequency measured in the experiment. It is clearly seen that the secondary peak frequency is three times the excitation frequency for both pipes. In other words, the measurements match well with the theoretical values. Although the Strouhal is only strictly defined for stationary cylinders, it is used here to describe a relationship between the observed response frequency and the flow speed. The Strouhal number, the diameter, the excitation frequency and the flow speed satisfy the relationship $f=S_{t} U / D$. Thus, the Strouhal numbers for the bare medium pipe and the bare large pipe are determined by a linear best fit straight line. They observed values are 0.130 for the bare medium pipe and 0.136 for the bare large pipe.

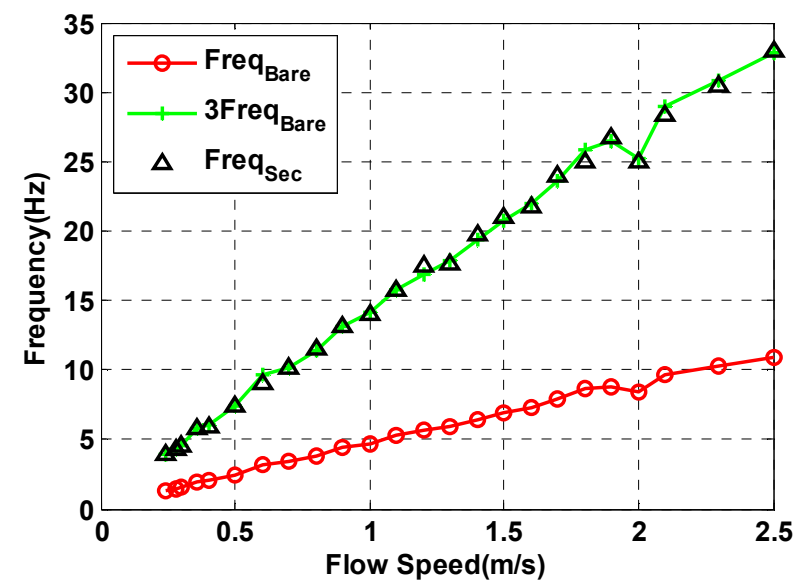

Figure 3. Bare excitation (Freq $\mathrm{Bare}$ ) and secondary frequencies $\left(\right.$ Freq $\left._{\mathrm{Sec}}\right)$ are plotted against flow speed for uniform flow for the bare medium pipe $(D=30 \mathrm{~mm})$. The green line represents the theoretical value of three times of the excitation frequency for bare medium pipe and black triangle represents the secondary peak frequency measured in the experiment. 


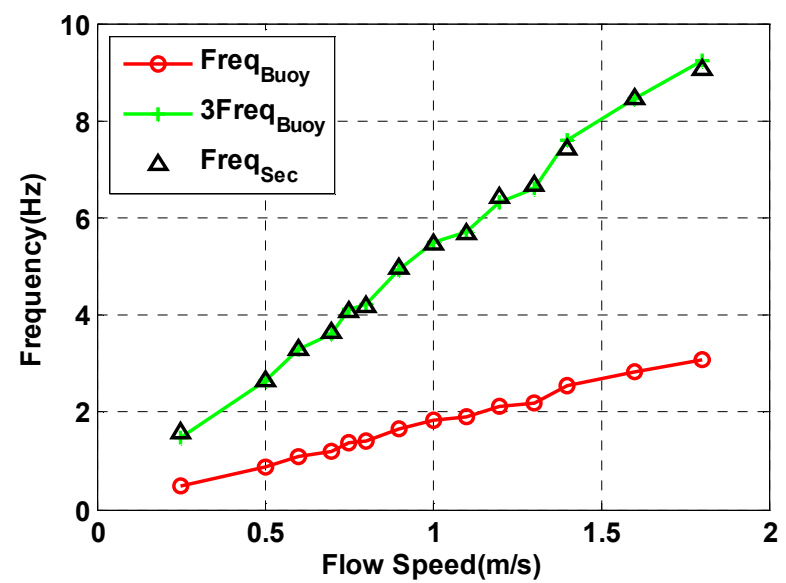

Figure 4. Buoyancy excitation (Freq Buoy $_{\text {) }}$ ) and secondary frequencies $\left(\right.$ Freq $\left.\mathrm{Sec}_{\mathrm{c}}\right)$ are plotted against flow speed for uniform flow for the bare large pipe $(D=80 \mathrm{~mm})$. The green line represents the theoretical value of three times of the excitation frequency for bare large pipe and black triangle represents the secondary peak frequency measured in the experiment.

The reason for the existence of the odd multiples of the excitation for bare pipes under uniform flow can be explained as follows.

For a bare pipe under uniform flow, the whole length of the pipe is the power in region and the damping term, $c(x, t)$, only accounts for the structural damping but no hydrodynamic damping. Because the structural system is linear, the frequency components in the VIV response would be exactly the same as those in the excitation force. The excitation force is periodic but not purely sinusoidal because the lift coefficient is not constant but a function of VIV displacement. If the periodic excitation force is decomposed into a Fourier series, odd multiples of the fundamental periodic excitation frequency would be observed. In other words, a non-sinusoidal periodic function has additional frequency components at three and five times the fundamental excitation frequency. The response of a linear system to this excitation would include the higher frequency components.

\section{Frequency components in the pipe with buoyancy modules}

For a flexible cylinder with staggered buoyancy modules, it is expected that two different frequencies are excited. The bare region with the smaller diameter is always excited at the higher frequency, and the buoyant region with the larger diameter is always excited at the lower frequency.

Figure 5 shows an example curvature power spectrum of the medium pipe with staggered buoyancy modules. The data is the averaged spectrum of 30 curvature sensors along the pipe's span at $1.0 \mathrm{~m} / \mathrm{s}$ uniform flow, corresponding to Test 6067 with
$L_{c} / L_{b}=2 / 2$. In Figure 5, a third frequency is observed. This is the non-linear interaction frequency predicted early.

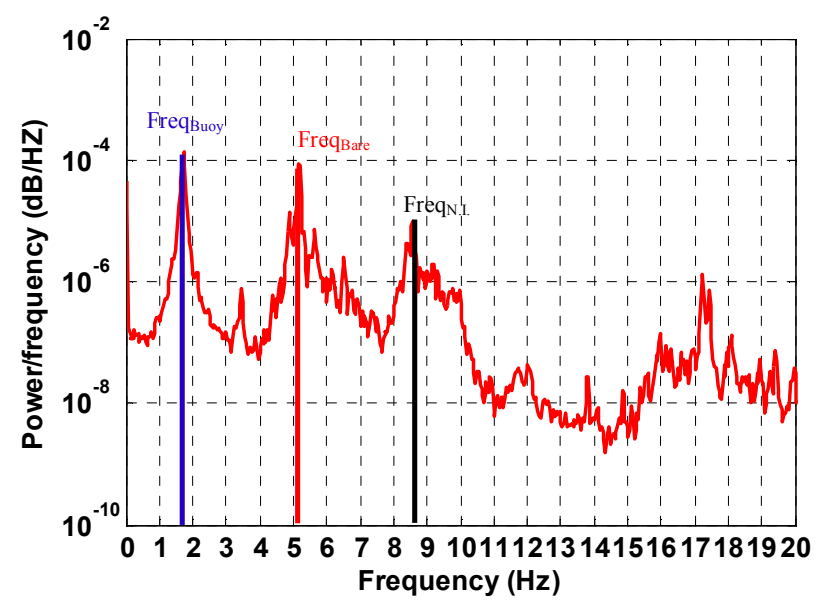

Figure 5. An example curvature power spectrum for the medium pipe with staggered buoyancy modules. The data is the averaged spectrum of 30 curvatures at $1.0 \mathrm{~m} / \mathrm{s}$ uniform flow, corresponding to Test 6067 with $L_{c} / L_{b}=2 / 2$.

The third frequency is also called the "non-linear interaction

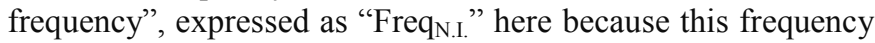
is due to the nonlinear interaction of the response of the cylinder at two excitation frequencies associated with bare and buoyant regions, which will be discussed later. Figures 6 to 10 show the two excitation frequencies, "Freq Bare" and "Freq

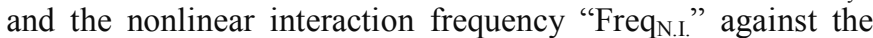
flow speed for five buoyancy configurations. Here, "Freq Bare" was the excitation frequency associated with smaller diameter

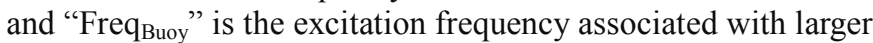
diameter. Similar to Figures 3 and 4, the green line in Figures 6 through 10 represents the theoretical value of the sum of the frequency associated with bare region and twice of the frequency associated with buoyant region. The black triangle represents the nonlinear interaction frequency measured in the experiment. Figures 6 to 10 show that the nonlinear interaction

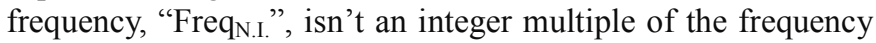
associated with either bare or buoyant regions, but the sum of the frequency associated with the bare region and twice the frequency associated with buoyant region, $2 \mathrm{Freq}_{\mathrm{Buoy}}+\mathrm{Freq}_{\mathrm{Bare}}$. This new observation has not been previously reported and requires explanation. 


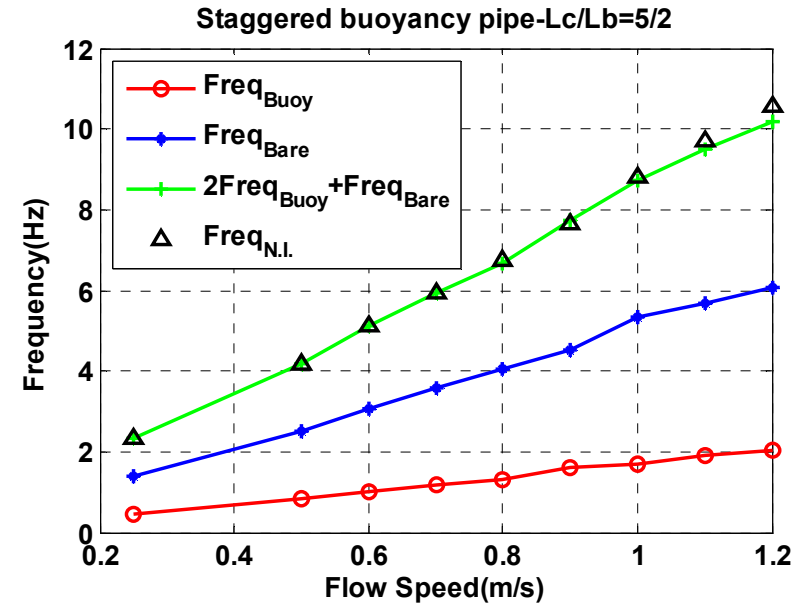

Figure 6. Buoyancy excitation frequency, bare excitation frequency and nonlinear interaction frequency in the curvature power spectrum are plotted against flow speed. The data is the averaged spectrum of 30 curvatures, corresponding to the buoyancy configuration $L_{c} / L_{b}=5 / 2$. The green line represents the theoretical value of $2 \mathrm{Freq}_{\mathrm{Buoy}}+$ Freq $\mathrm{Bare}_{\mathrm{B}}$ and black triangle represents the nonlinear interaction frequency measured in the experiment.

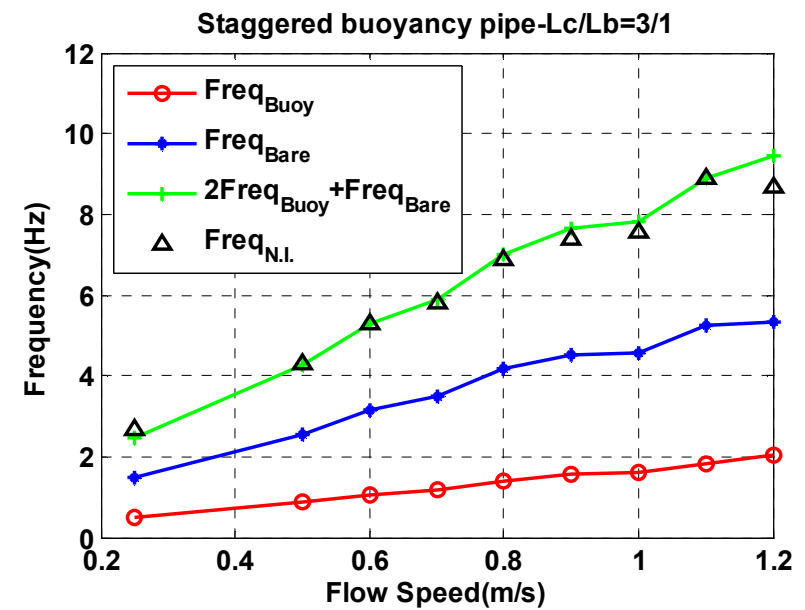

Figure 7. Buoyancy excitation frequency, bare excitation frequency and nonlinear interaction frequency in the curvature power spectrum are plotted against flow speed. The data is the averaged spectrum of 30 curvatures, corresponding to the buoyancy configuration $L_{c} / L_{b}=3 / 1$. The green line represents the theoretical value of $2 \mathrm{Freq}_{\mathrm{Buoy}}+\mathrm{Freq}_{\mathrm{Bare}}$ and black triangle represents the nonlinear interaction frequency measured in the experiment.

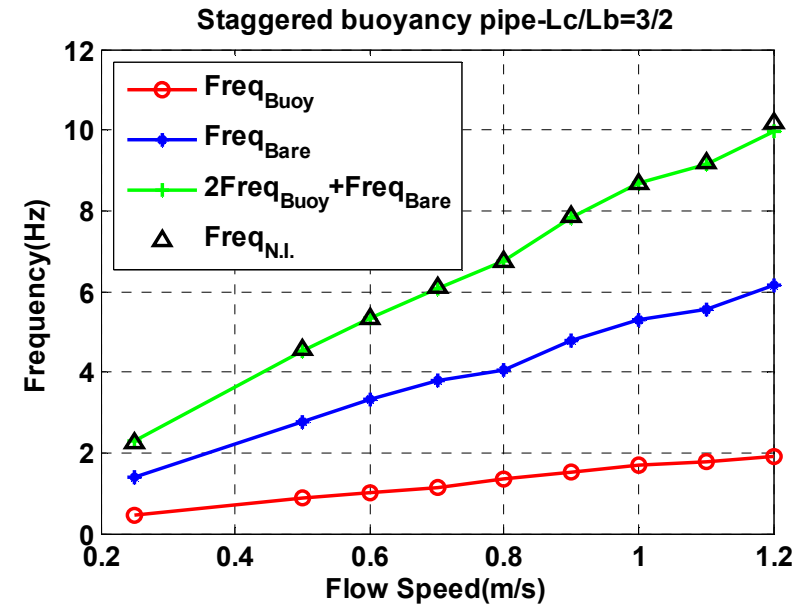

Figure 8. Buoyancy excitation frequency, bare excitation frequency and nonlinear interaction frequency in the curvature power spectrum are plotted against flow speed. The data is the averaged spectrum of 30 curvatures, corresponding to the buoyancy configuration $L_{c} / L_{b}=3 / 2$. The green line represents the theoretical value of $2 \mathrm{Freq}_{\mathrm{Buoy}}+\mathrm{Freq}_{\mathrm{Bare}}$ and black triangle represents the nonlinear interaction frequency measured in the experiment.

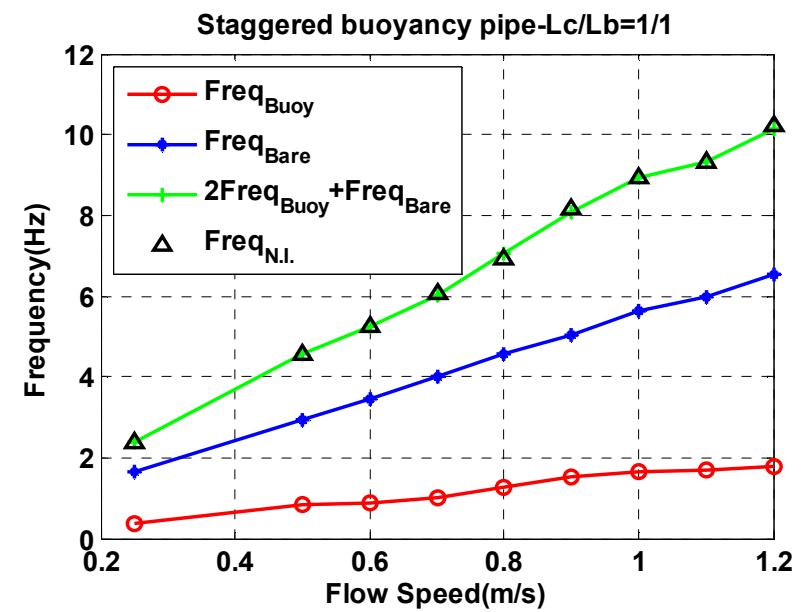

Figure 9. Buoyancy excitation frequency, bare excitation frequency and nonlinear interaction frequency in the curvature power spectrum are plotted against flow speed. The data is the averaged spectrum of 30 curvatures, corresponding to the buoyancy configuration $L_{c} / L_{b}=1 / 1$. The green line represents the theoretical value of $2 \mathrm{Freq}_{\mathrm{Buoy}}+\mathrm{Freq}_{\mathrm{Bare}}$ and black triangle represents the nonlinear interaction frequency measured in the experiment. 


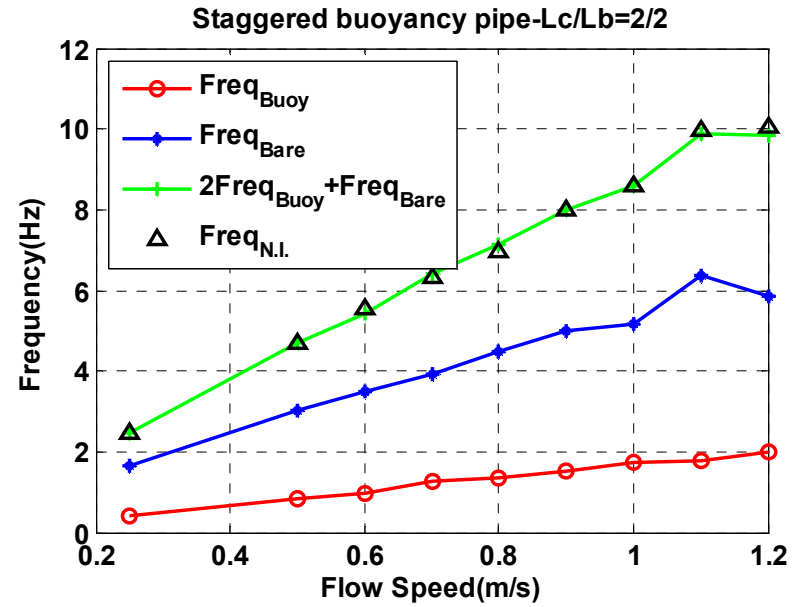

Figure 10. Buoyancy excitation frequency, bare excitation frequency and nonlinear interaction frequency in the curvature power spectrum are plotted against flow speed. The data is the averaged spectrum of 30 curvatures, corresponding to the buoyancy configuration $L_{c} / L_{b}=2 / 2$. The green line represents the theoretical value of $2 \mathrm{Freq}_{\mathrm{Buoy}}+$ Freq $\mathrm{Bare}_{\mathrm{B}}$ and black triangle represents the nonlinear interaction frequency measured in the experiment.

A possible explanation for the existence of the nonlinear interaction frequency may be due to the nonlinearity of the hydrodynamic damping mechanism.

For a pipe with staggered buoyancy modules, the bare region is the power-in region at the higher excitation frequency while the buoyant region is the power-in region at the lower excitation frequency. The damping in the governing equation is no longer constant due to the existence of the hydrodynamic damping. And the structural system is no longer linear because the damping and amplitude influence each other. The excitation forces are at two main excitation frequencies. One is associated with the excitation on bare regions and the other is associated with the excitation on buoyant regions. The authors derived the potential nonlinear interaction frequencies in theory for the pipe with two excitation frequencies. At this time, the authors cannot explain why the nonlinear interaction frequency favored 2 Freq $_{\mathrm{Buoy}}+\mathrm{Freq}_{\mathrm{Bare}}$, but not other possible combinations of the two excitation frequencies, as predicted by theory. The nonlinear interaction between the responses of the cylinder at two excitation frequencies exists only when the frequency and its wavenumber meet the dispersion relation. For the pipe with staggered buoyancy modules, the dispersion relation is not obvious due to the non-uniform distribution of mass.

Figures 11 and 12 summarize the excitation frequencies associated with the larger and smaller diameters, respectively. The excitation frequency is linearly related to the flow speed. The lines closely correspond to vortex shedding controlled by two different diameters. The Strouhal number (not strictly defined) is defined as the diameter multiplied by the frequency of vortex shedding divided by the flow speed. The observed Strouhal numbers for bare pipe and pipe with staggered buoyancy are summarized in the Table 2.

Table 2: The summary of the St Number for pipes

\begin{tabular}{|c|c|c|}
\hline Type of the pipe & St for $\mathrm{D}=30 \mathrm{~mm}$ & St for $\mathrm{D}=80 \mathrm{~mm}$ \\
\hline Pipe $2 /$ Pipe 3 & 0.136 & 0.130 \\
\hline $\mathrm{Lc} / \mathrm{Lb}=5 / 2$ & 0.153 & 0.136 \\
\hline $\mathrm{Lc} / \mathrm{Lb}=3 / 1$ & 0.125 & 0.129 \\
\hline $\mathrm{Lc} / \mathrm{Lb}=3 / 2$ & 0.148 & 0.125 \\
\hline $\mathrm{Lc} / \mathrm{Lb}=1 / 1$ & 0.155 & 0.125 \\
\hline $\mathrm{Lc} / \mathrm{Lb}=2 / 2$ & 0.143 & 0.132 \\
\hline
\end{tabular}

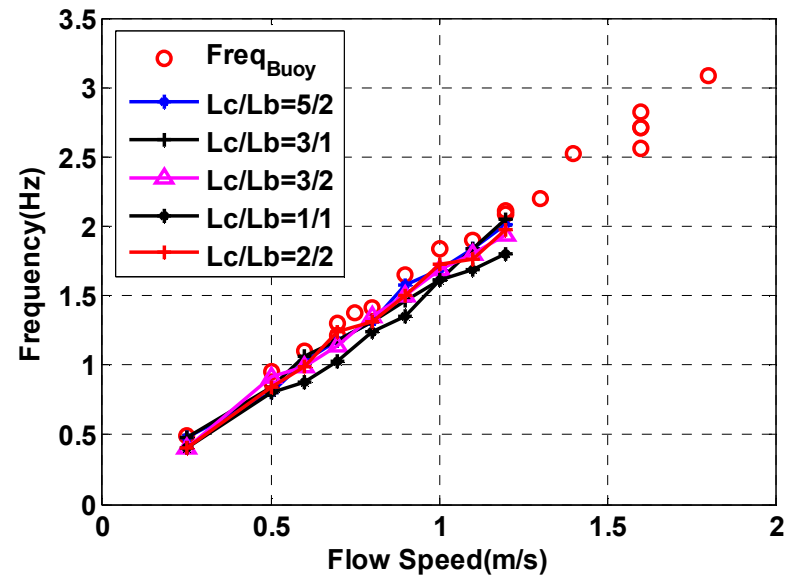

Figure 11. Excitation frequencies associated with larger diameter in both bare large pipe (red circle) and five buoyancy configurations (line+symbols) are plotted against flow speed.



Figure 12. Excitation frequencies associated with smaller diameter in both bare medium pipe (red circle) and five buoyancy configurations (line+symbols) are plotted against flow speed.

\section{The effect of the nonlinear interaction frequency on VIV}

A new phenomenon was observed in the pipe with staggered buoyancy modules; the nonlinear interaction frequency isn't an integer multiple of the frequency associated with bare or buoyancy regions, but the sum of the frequency associated with 
bare regions plus twice the frequency associated with buoyancy regions. It is important to determine if response at this interaction frequency is important in terms of response amplitude and in terms of damage rate. In order to determine the contribution to total response that comes from the interaction term, the response amplitude must be determined from the acceleration and strain data obtained in these experiments. In this study response amplitude is estimated by the method of modal response reconstruction based on a $2 \mathrm{D}$ FFT technique, as described in Rao et al ${ }^{[12]}$. This method requires orthogonal vectors to represent the contributions of each mode. Two methods were used to obtain orthogonal mode shapes in this study. The first was to use simple sinusoidal functions. The second was to use a finite element model to obtain mode shapes, which are also orthogonal vectors. However, the mode shapes determined by the FEM are affected by the non-uniform mass distribution associated with staggered buoyancy modules. The non-uniformly distributed mass results in the non-sinusoidal mode shapes.

It is shown below that reconstructed displacements based on sinusoidal or real(FEM) mode shapes yield the same results. Here, the real mode shapes of the pipe with staggered buoyancy were obtained with ANSYS. The comparison is shown in Figure 13. The red line represents the reconstructed response with real mode shapes and the blue line represents the reconstructed displacement accomplished with sinusoidal mode shapes. The figures on the left and right show the comparison of the reconstructed displacement with sinusoidal and real mode shapes at the higher and lower excitation frequencies, respectively. These two reconstructed displacements are nearly identical. Although this example was for the case of $L_{c} / L_{b}=2 / 2$, the results are similar for other cases tested.

In the example shown in Figure 17 the response to excitation on the buoyancy modules was largely $3^{\text {rd }}$ mode. Thus there were approximately 15 buoyancy modules per half wavelength of the mode shape. For the figure on the left the dominant response mode is the $11^{\text {th }}$ and the number of modules per half wavelength is approximately 4 . The greater the number of modules per half wavelength, the less the modules would be expected to locally alter the mode shape from that of a uniform cylinder.

Because it was found that the type of mode shape used did not affect the estimated VIV response amplitude, sinusoidal mode shapes were used for a remaining cases in the paper.
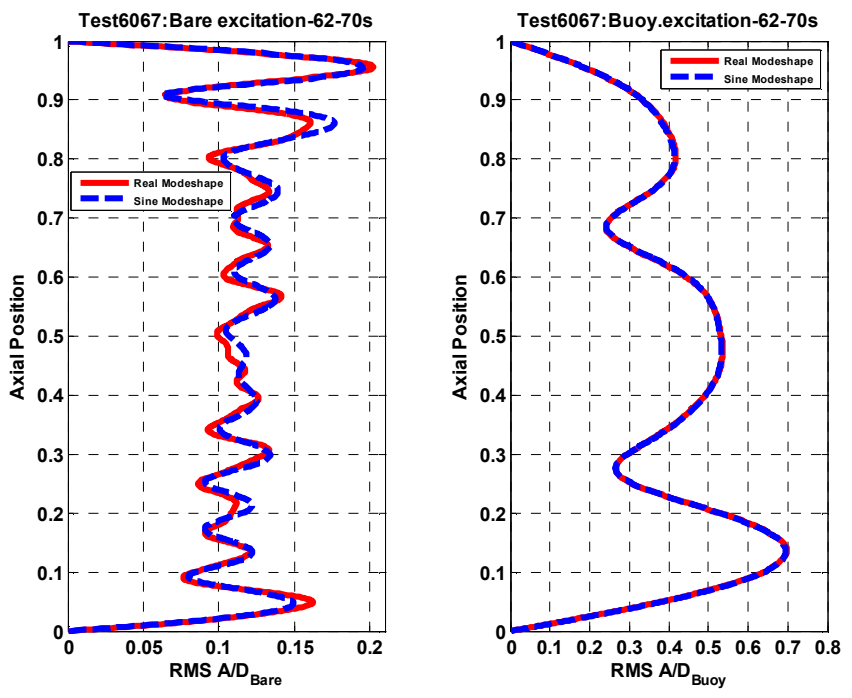

Figure 13. An example comparison of reconstructed displacements with sinusoidal and real mode shapes. The data is from Test 6067 with $L_{c} / L_{b}=2 / 2$ at $1.0 \mathrm{~m} / \mathrm{s}$ uniform flow. Band-pass filter in frequency for the bare excitation and the buoyancy excitation is from $0.5 \mathrm{x}$ Freq $\mathrm{Bare}_{\mathrm{B}}$ to

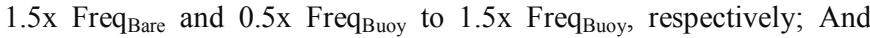
filter bandpass values are $\pi / L$ to $29 \pi / L$ in wave number for both excitations.

Figure 14 shows the ratio of the maximum RMS displacement due to the excitation at the nonlinear interaction frequency $\left(A_{N . I}\right)$ and the maximum RMS displacement $\left(A_{\text {total }}\right)$ versus flow speed. The contribution of the excitation at the nonlinear interaction frequency to the total VIV displacement is less than $5 \%$ for most cases. The contribution of the maximum damage rate with rainflow method [13] at the nonlinear interaction frequency was also checked. Most cases are below $8 \%$ and 3 out of 45 cases are close to $20 \%$.

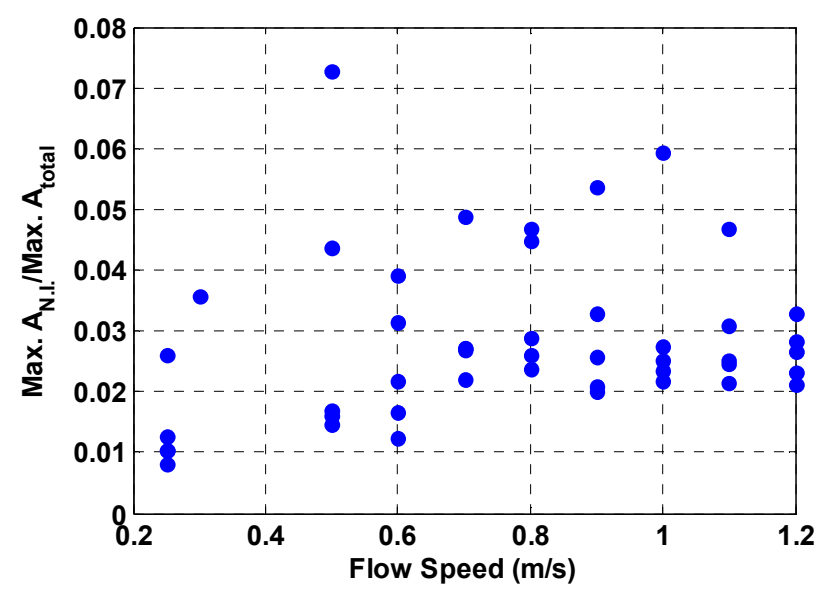

Figure 14. The ratio of the maximum RMS displacement due to the excitation at the nonlinear interaction frequency to the maximum RMS displacement versus flow speed. 


\section{VIV power estimation at the excitation frequency}

In this paper, power extracted from the flow will be used as the metric which determines whether bare or buoyant regions are dominant. It is found that there are three main excitation frequencies in the curvature power spectrum. The contribution of the excitation at the nonlinear interaction frequency on the total VIV response is small. Thus the excitation competition between bare and buoyant regions becomes the competition of the excitation at two excitation frequencies.

Here, the excitation competition is expressed as the ratio of the power dissipated by damping at the higher frequency to that dissipated at the lower frequency. When the power ratio is greater than one, it suggests that the excitation on bare regions is dominant. Vice versa, when the power ratio is smaller than one, it suggests that the excitation on buoyant regions is dominant.

The excitations on bare and buoyant regions could be identified through the frequency components. The average power due to the excitation on bare and buoyant regions could be computed numerically with Eqs. (6) and (7)

$$
\begin{gathered}
P_{\text {bare }}=\int_{L} \frac{1}{T} \int_{0}^{T} c_{\text {bare }} \dot{y}_{\text {bare }}^{2} d t d x \\
P_{\text {buoyancy }}=\int_{L} \frac{1}{T} \int_{0}^{T} c_{\text {buoyancy }} \dot{y}_{\text {buoyancy }}^{2} d t d x
\end{gathered}
$$

Where the subscripts "bare" and "buoyancy" represent the excitation associated with smaller and larger diameters, respectively. For example, $P_{\text {bare }}$ and $P_{\text {buoyancy }}$ represent the average power at higher and lower excitation frequency, respectively. Similarly, the reconstructed displacement $y_{\text {bare }}$ and $y_{\text {buoyancy, }}$, the damping $c_{\text {bare }}$ and $c_{\text {buovancy }}$ represent the corresponding values at the higher and lower frequency, respectively. When the response is excited at the higher frequency, the bare region is the power-in region and the buoyant region becomes the power-out region. In the power-in region, $c_{\text {bare }}$ is only due to the structural damping. In the powerout region, $c_{\text {bare }}$ includes both structural and hydrodynamic damping. Vice versa, when the response is excited at the lower frequency, the buoyant region now becomes the power-in region and the bare region is the power-out region. In the power-in region, $c_{\text {buoyancy }}$ only has the structural damping and no hydrodynamic damping; in the power-out region, $c_{\text {buovancy }}$ includes both structural and hydrodynamic damping. Here, the Venugopal's hydrodynamic damping model is used to compute the hydrodynamic damping in the power-out region.

Figures 15 and 16 show the average VIV power due to the excitation on bare and buoyant regions for different buoyancy configurations, respectively. " $5 / 2$ " stands for $L_{c} / L_{b}=5 / 2$ where
$L_{b}$ is the length of the buoyant segment and $L_{c}$ the gap between two adjacent buoyant segments. It is to be expected that the power increases with the flow speed for all five buoyancy configurations at both excitation frequencies.

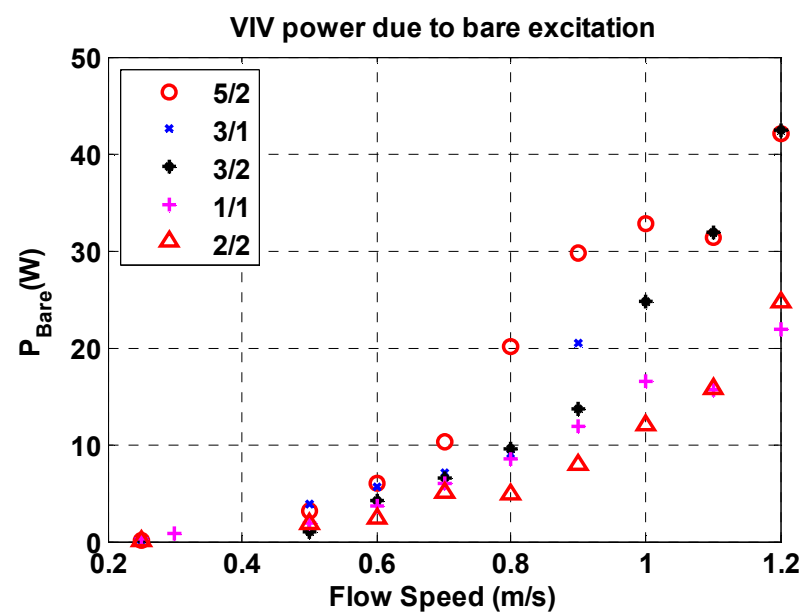

Figure 15. VIV power due to the excitation on bare regions versus flow speed

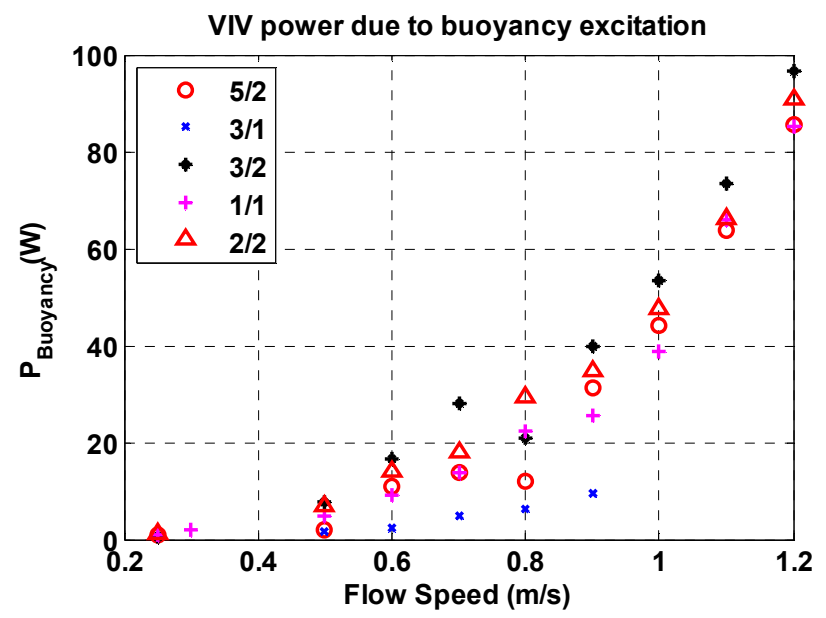

Figure 16. VIV power due to the excitation on buoyancy regions versus flow speed

Figure 17 shows the ratio of the power dissipated by damping at the higher frequency and that at the lower frequency, $P_{\text {bare }} / P_{\text {buovancy }}$. When the ratio is greater than one, it suggests the excitation on bare regions dominates the response; Vice versa, when the power ratio is smaller than one, it suggests that the excitation on buoyant regions is dominant. From Figure 17, it is found that the excitation on bare regions dominates the response for the buoyancy configuration $L_{c} / L_{b}=3 / 1$ at almost all flow speeds and the buoyancy configuration $L_{c} / L_{b}=5 / 2$ at some flow speeds. While the excitation on buoyancy regions dominates the response for the buoyancy configurations $L_{c} / L_{b}=3 / 2, L_{c} / L_{b}=1 / 1, L_{c} / L_{b}=2 / 2$ at all flow speeds and the buoyancy configuration $L_{c} / L_{b}=5 / 2$ at most flow speeds. The data analysis shows that the coverage of buoyancy modules 
play a major role in the excitation competition between bare and buoyant regions.

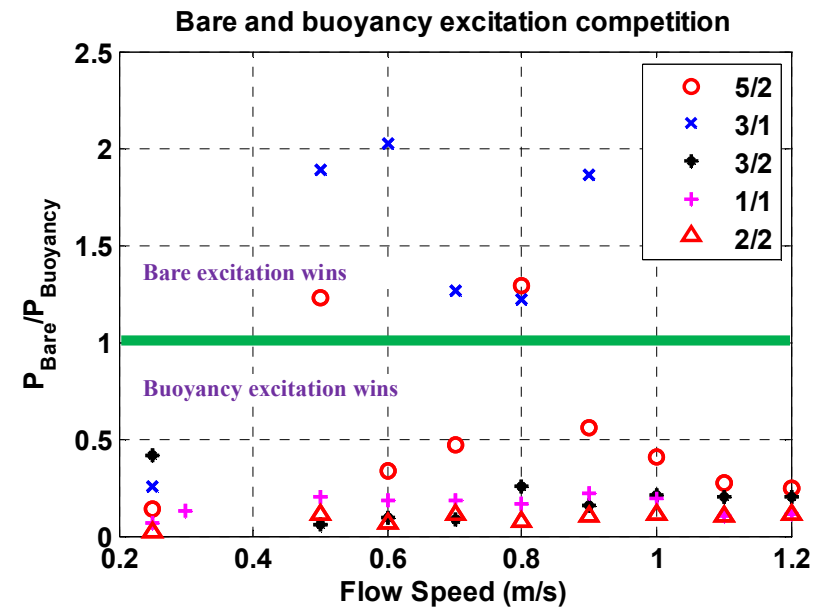

Figure 17. The ratio of the VIV power due to the excitation on bare regions and the VIV power due to the excitation on buoyant regions

\section{A predictor for excitation competition}

In order to predict the winner of the excitation competition between bare and buoyant segments for given buoyancy coverage, a simplified expression is derived as a reference based on some assumptions. For example, the pipe with staggered buoyancy modules is split into two separated pipes. One has the same diameter and length as bare regions in the pipe with staggered buoyancy modules and the other has the same diameter and length as buoyant regions in the pipe with staggered buoyancy modules.

Suppose the lift force per unit length and the VIV displacement are given by:

$$
F=\frac{1}{2} C_{L} \rho D U^{2} \sin k x \cos \omega t, y=A \sin k x \sin \omega t
$$

The predicted power due to the excitation associated with smaller diameter is given:

$$
\overline{P_{\text {bare }}}=\int_{L_{\text {bare }}} \frac{1}{T} \int_{0}^{T} F_{\text {bare }} \dot{y}_{\text {bare }} d t d x=\frac{1}{8} \rho\left(C_{L} D U^{2} A \omega L\right)_{b a r e}
$$

The predicted power due to the excitation associated with larger diameter is given:

$$
\overline{P_{\text {buoyancy }}}=\int_{L_{\text {buoyancy }}} \frac{1}{T} \int_{0}^{T} F_{\text {buoyancy }} \dot{y}_{\text {buoyancy }} d t d x
$$

$$
=\frac{1}{8} \rho\left(C_{L} D U^{2} A \omega L\right)_{\text {buoyancy }}
$$

The excitation competition is expressed as the ratio of the VIV power on bare regions to the VIV power on buoyant regions.

$$
\frac{\overline{P_{\text {bare }}}}{\overline{P_{\text {buoyancy }}}}=\frac{\left(C_{L} D U^{2} A \omega L\right)_{\text {bare }}}{\left(C_{L} D U^{2} A \omega L\right)_{\text {buoyancy }}}
$$

Since the entire pipe is exposed to a uniform current profile, $U_{\text {bare }}=U_{\text {buoyancy. }}$.

Here, we assume that the VIV response for the equivalent bare pipe and buoyant pipe have the same non-dimensional amplitude $\frac{A_{\text {bare }}}{D_{\text {bare }}}=\frac{A_{\text {buoyancy }}}{D_{\text {buoyancy }}}$ based on the reason that when the excitation frequency is close to the natural frequency of the pipe and the VIV is at lock-in, it will achieves the maximum amplitude. According to the lift coefficient curve, the lift coefficient at the same non-dimensional amplitude and frequency would be the same for both pipes, namely $C_{\text {Lbare }}=$ $C_{\text {Lbuoyancy. }}$ The excitation competition is expressed as follows:

$$
\frac{\overline{P_{\text {bare }}}}{\overline{P_{\text {buoyancy }}}}=\frac{D_{\text {bare }}}{D_{\text {buoyancy }}} \frac{L_{\text {bare }}}{L_{\text {buoyancy }}}
$$

If the lift force magnitude ratio is the metric used to determine the winner of the excitation competition, the expression will be the same as Eq.(12), $\frac{D_{\text {bare }}}{D_{\text {buoyancy }}} \frac{L_{\text {bare }}}{L_{\text {buoyancy }}}$ which can be seen in Lie et $\mathrm{al}^{[1]}$. If the amplitude ratio of dominant modes is the metric used to determine the winner of the excitation competition, the expression will be $\left(\frac{D_{\text {bare }}}{D_{\text {buoyancy }}} \frac{L_{\text {bare }}}{L_{\text {buoyancy }}}\right)^{2}$, which can be seen in Vandiver [5].

Equations $8 \& 9$ assumed standing wave excitation and response. If travelling wave excitation and response were considered, the result in equation 12 would be the same.

All these expressions suggest that when the ratio in Eq.12 is greater than one, the excitation on bare regions dominate; and when this ratio is less than one, the excitation on buoyant regions dominate. The prediction in Eq.(12) is relatively rough because it doesn't consider the effect of the placement of the staggered buoyancy or Reynolds number. But it may be used in predicting the actual behavior for the pipe with staggered buoyancy modules. Next, we present data from experiments to test this predictor.

Table 3 lists the comparison of the excitation competition with the expression in Eq.(12) and experimental results according to Section 4. The comparison results show that the prediction results match remarkably with the experiment results. Here 
$\frac{D_{\text {bare }}}{D_{\text {buoyancy }}}=\frac{3}{8}$ for the SHELL test and $\frac{\overline{P_{\text {bare }}}}{\overline{P_{\text {buoyancy }}}}$ in the Table 3 was obtained with Eq.(12). "Buoyancy" and "Bare" in Tables 3 and 4 mean the excitation on buoyant or bare regions dominates, respectively.

Table 3: the winner of the excitation competition for SHELL Test

\begin{tabular}{|c|c|c|c|}
\hline$\frac{L_{\text {bare }}}{L_{\text {buoyancy }}}$ & $\overline{\overline{P_{\text {bare }}}}$ & $\begin{array}{c}\text { Prediction } \\
P_{\text {buoyancy }}\end{array}$ & $\begin{array}{c}\text { Measurement } \\
\text { Eq.(12)/Eq.(9) }\end{array}$ \\
\hline $5 / 2$ & 0.9375 & Buoyancy & $\begin{array}{c}\text { Buoyancy(most cases), } \\
\text { Bare (few cases) }\end{array}$ \\
\hline $3 / 1$ & 1.125 & Bare & Bare \\
\hline $3 / 2$ & 0.5625 & Buoyancy & Buoyancy \\
\hline $1 / 1$ & 0.375 & Buoyancy & Buoyancy \\
\hline $2 / 2$ & 0.375 & Buoyancy & Buoyancy \\
\hline
\end{tabular}

Comparing the predictor in the $3^{\text {rd }}$ column with the actual winner of the excitation competition $\left(4^{\text {th }}\right.$ column), it shows that the predictor is capable of giving considerable insight as to which excitation will dominate the VIV response for the pipe with staggered buoyancy modules under uniform flow. And it also shows that the excitation on the buoyant region is favored to dominate the response due to its larger diameter.

Vandiver and Peoples [2] also studied the excitation competition in a similar VIV experiment. The frequency of the largest spectral peak was used to determine the winner of the excitation competition between bare and buoyant segments. In their experiment, the diameter of bare pipe was $20 \mathrm{~mm}$ and that of buoyancy modules $50 \mathrm{~mm}$. Four buoyancy configurations, $100 \%$ coverage, $50 \%$ coverage $25 \%$ coverage and $0 \%$ coverage of buoyancy modules, were studied. The detailed results are listed in Table 4. The comparison results also show that the predicted results matched as expected with the experimental results.

Table 4: the winner of the excitation competition in Vandiver and Peoples

\begin{tabular}{|c|c|c|c|}
\hline Coverage & $\frac{\overline{P_{\text {bare }}}}{\overline{P_{\text {buoyancy }}}}$ & $\begin{array}{c}\text { Prediction } \\
\text { Eq.(12) }\end{array}$ & $\begin{array}{c}\text { Results from } \\
\text { Vandiver and Peoples }\end{array}$ \\
\hline $100 \%$ & 0 & Buoyancy & Buoyancy \\
\hline $50 \%$ & 0.4 & Buoyancy & Buoyancy \\
\hline $25 \%$ & 1.2 & Bare & $\begin{array}{c}\text { Bare (most cases) } \\
\text { Buoyancy (few cases) }\end{array}$ \\
\hline $0 \%$ & - & Bare & Bare \\
\hline
\end{tabular}

\section{CONCLUSIONS}

The primary contribution of this work was to explore the excitation competition between bare and buoyant segments under uniform flows.
A summary of observations and preliminary conclusions from the VIV analysis of the pipe with staggered buoyancy modules under uniform flows includes:

(1) A new phenomenon was observed. The nonlinear interaction frequency in the staggered buoyancy pipe is found not to be a multiple of the frequency associated with either bare or buoyant regions, but the sum of the frequency associated with bare region and twice of the frequency associated with buoyant region. The reason may be the nonlinearity of the hydrodynamic damping. All cases studied were for uniformly distributed buoyancy modules. It is not known if the nonlinear interaction frequency would appear if, for example, all the buoyancy was placed on one end of the riser and all the bare was at the other end. Resolving this requires additional research.

(2) The contribution of the excitation at the nonlinear interaction frequency to the total VIV amplitude is less than $5 \%$ for most cases. Thus the effect of the excitation at this nonlinear interaction frequency on the total VIV response is small.

(3) The power ratio is the metric used to determine the winner of excitation competition in the SHELL tests. It is defined as $\frac{P_{\text {bare }}}{P_{\text {buoyancy }}}$. When the power ratio is greater than one, it suggests that the excitation on bare regions will be dominant. Vice versa, when the power ratio is smaller than one, it suggests that the excitation on buoyant regions will be dominant.

(4) Excitation competition results in the SHELL tests show that for most buoyancy configurations except $L_{c} / L_{b}=3 / 1$, the excitation on buoyant regions dominates the VIV response.

(5) The expression $\frac{\overline{P_{\text {bare }}}}{\overline{P_{\text {buoyancy }}}}=\frac{D_{\text {bare }}}{D_{\text {buoyancy }}} \frac{L_{\text {bare }}}{L_{\text {buoyancy }}}$ is proposed to predict the winner of the excitation competition for a pipe with staggered buoyancy modules under uniform flow. The prediction matches well with the experimental data. It may be used in predicting the actual behavior for pipe with staggered buoyancy modules.

In terms of the fatigue damage rate, more research is required to determine whether dominance by buoyant or bare regions is desirable. For now that must be determined on a case by case basis using a response prediction program such as Shear7.

The next step in analysis of this data is to construct a plot similar to figure 17 which companies the damage rates from the measured data to see which distribution of buoyancy modules is preferable. 


\section{ACKNOWLEDGMENTS}

The authors gratefully acknowledge Deepstar and the SHEAR7 JIP members (BP, Chevron, ExxonMobil, Petrobras, Shell, Statoil \& Technip) for supporting this research, and especially SHELL Exploration and Production for providing the data. We also thank Mr.Themistocles L.Resvanis of MIT for valuable discussions and revisions.

\section{REFERENCES}

[1] Halvor Lie, Knut Mo and J.Kim Vandiver (1998). VIV model test of a bare and a staggered buoyancy riser in a rotating rig. OTC 8700.

[2] J.Kim Vandiver and W.Wesley Peoples (2003). The effect of staggered buoyancy modules on flow-induced vibration of marine. OTC15284.

[3] Lin Li, Shixiao Fu, Jianmin Yang, Tie Ren and Xuefeng Wang (2011). Experimental investigation on vortexinduced vibration of risers with staggered buoyancy. OMAE2011-49046.

[4] Vikas Jhingran, Heping Zhang, Halvor Lie, Henning Bratton and J.Kim Vandiver (2012). Buoyancy spacing implications for fatigue damage due to vortex-induced vibrations on a steel lazy wave riser (SLWR). OTC23672

[5] J.Kim Vandiver (2000). Predicting lock-in on drilling risers in sheared flows. The Proceedings of the Flow-Induced Vibration 2000 Conference, Lucerne, Switzerland. June 1822, 2000.

[6] R.H Enns, S.S. Rangnekar (1979). The three-wave interaction in nonlinear optics. Journal of Physica Status Solidi(b).

[7] A.D.D. CRAIK (1985). Wave interactions and fluid flows. Cambridge University Press.

[8] P.P. Banerjee and A.Korpel (1982). Subharmonic generation by resonant three-wave interaction of deep-water capillary waves. Journal of Physics of Fluids.

[9] F. P. Bretherton (1964). Resonant interactions between waves. The case of discrete oscillations. Journal of Fluid Mechanics.

[10] Venugopal, M., 1996, "Damping and Response of a Flexible Cylinder in Current", PhD thesis, Department of Ocean Engineering, Massachusetts Institute of Technology, Cambridge, USA.

[11] J.Kim Vandiver and T-Y.Chung (1988). Predicted and measured response of flexible cylinders in sheared flow. Proc. ASME Winter Annual Meeting Symposium on FlowInduced Vibration, Chicago, December 1988.

[12] Zhibiao Rao, J.Kim Vandiver, Vikas Jhingran and Octavio Sequeiros (2012). The effect of exposure length on vortex induced vibration of flexible cylinders. OMAE2012-83273.

[13] J.K.Vandiver, S.B.Swithenbank, V.Jaiswal and V.Jhingran (2006). Fatigue damage from high mode number vortexinduced vibration. OMAE2006-9240.

\section{Appendix}

\section{Asymptotic expansion}

This appendix is trying to find the possible frequency components in the response for a nonlinear motion equation.

For a flexible cylinder with staggered buoyancy, the excitation forces are at two different frequencies. In more general, the excitation force at a frequency may be also in a form of traveling wave with two directions. Thus, the excitation force could be expressed as:

$$
\begin{gathered}
F(x, t)=F_{1} e^{j\left(k_{1} x-\omega_{1} t\right)}+F_{2} e^{j\left(k_{1} x+\omega_{1} t\right)}+F_{3} e^{j\left(k_{2} x-\omega_{2} t\right)}+ \\
F_{4} e^{j\left(k_{2} x+\omega_{2} t\right)}
\end{gathered}
$$

In order to study the nonlinear interaction, all quantities are non-dimensionalized using appropriate combinations of $A, L, m, \omega_{n}$. The parameters are non-dimensionalized in the following way:

$\tau=\omega_{n} t, \bar{y}=\frac{y}{A}, \bar{x}=\frac{x}{L}, \quad 2 \epsilon=\frac{c_{1}}{m \omega_{n}}, 2 \epsilon \beta=\frac{c_{2} a^{2}}{m \omega_{n}}, \lambda_{1}=\frac{E I}{m \omega_{n}^{2}} \frac{1}{L^{4}}$,

$\lambda_{2}=\frac{T}{m \omega_{n}^{2}} \frac{1}{L^{2}}$

Suppose the excitation forces are at the same order of the damping forces.

$\epsilon \overline{F_{1}}=\frac{F_{1}}{m \omega_{n}^{2} A}, \epsilon \overline{F_{2}}=\frac{F_{2}}{m \omega_{n}^{2} A}, \epsilon \overline{F_{3}}=\frac{F_{3}}{m \omega_{n}^{2} A}, \epsilon \overline{F_{4}}=\frac{F_{4}}{m \omega_{n}^{2} A}$

$\overline{k_{1}}=k_{1} L, \overline{k_{2}}=k_{2} L, \Omega_{1}=\frac{\omega_{1}}{\omega_{n}}, \Omega_{2}=\frac{\omega_{2}}{\omega_{n}}$

Substituting the Eq.(A.1) into the Eq.(1) and the new equation in dimensionless form is:

$$
\begin{aligned}
& \frac{d^{2} \bar{y}}{d \tau^{2}}+2 \epsilon \frac{d \bar{y}}{d \tau}+2 \epsilon \beta \overline{y^{2}} \frac{d \bar{y}}{d \tau}+\lambda_{1} \frac{d^{4} \bar{y}}{d \bar{x}^{4}}-\lambda_{2} \frac{d^{2} \bar{y}}{d \bar{x}^{2}}=\epsilon \overline{F_{1}} e^{j\left(\overline{k_{1}} \bar{x}-\Omega_{1} \tau\right)}+ \\
& \epsilon \overline{F_{2}} e^{j\left(\overline{k_{1}} \bar{x}+\Omega_{1} \tau\right)}+\epsilon \overline{F_{3}} e^{j\left(\overline{k_{2}} \bar{x}-\Omega_{2} \tau\right)}+\epsilon \overline{F_{4}} e^{j\left(\overline{k_{2}} \bar{x}+\Omega_{2} \tau\right)}
\end{aligned}
$$

\section{Represent}

$\bar{y}=u, \bar{x}=x, \quad \tau=t, \overline{k_{1}} \bar{x}-\Omega_{1} \tau=\theta_{1}, \overline{k_{1}} \bar{x}+\Omega_{1} \tau=\theta_{2}$, $\overline{k_{2}} \bar{x}-\Omega_{2} \tau=\theta_{3}, \overline{k_{2}} \bar{x}+\Omega_{2} \tau=\theta_{4}$

The vibration equation can be rewritten as:

$$
\begin{gathered}
\frac{d^{2} u}{d t^{2}}+2 \epsilon \frac{d u}{d t}+2 \epsilon \beta u^{2} \frac{d u}{d t}+\lambda_{1} \frac{d^{4} u}{d x^{4}}-\lambda_{2} \frac{d^{2} u}{d x^{2}}=\epsilon \overline{F_{1}} e^{j \theta_{1}}+ \\
\epsilon \overline{F_{2}} e^{j \theta_{2}}+\epsilon \overline{F_{3}} e^{j \theta_{3}}+\epsilon \overline{F_{4}} e^{j \theta_{4}}
\end{gathered}
$$


Here, we suppose the excitation frequencies $\Omega_{1}$ and $\Omega_{2}$ are resonant frequencies, and $\Omega_{1}=\omega_{5}+\sigma \epsilon, \Omega_{2}=\omega_{6}+\sigma \epsilon$, $\sigma \sim O(1)$. Here $\omega_{5}$ and $\omega_{6}$ are natural frequencies.

Due to $\epsilon$ is very smaller $(\epsilon \ll 1)$, the solution can be expressed in successive powers of $\epsilon$ by naïve expansion.

$$
u=u_{0}+\epsilon u_{1}+O\left(\epsilon^{2}\right)+\cdots
$$

Substituting this expansion into Eq.(A.3) and equating coefficients of like powers of $\boldsymbol{\epsilon}$, we obtain

$O(1)$

$$
\frac{d^{2} u_{0}}{d t^{2}}+\lambda_{1} \frac{d^{4} u_{0}}{d x^{4}}-\lambda_{2} \frac{d^{2} u_{0}}{d x^{2}}=0
$$

$\mathrm{O}(\boldsymbol{\epsilon})$

$$
\frac{d^{2} u_{1}}{d t^{2}}+\lambda_{1} \frac{d^{4} u_{1}}{d x^{4}}-\lambda_{2} \frac{d^{2} u_{1}}{d x^{2}}=-2 \frac{d u_{0}}{d t}-2 \beta u_{0}^{2} \frac{d u_{0}}{d t}+\Pi
$$

Where $\Pi=\overline{F_{1}} e^{j \theta_{1}}+\overline{F_{2}} e^{j \theta_{2}}+\overline{F_{3}} e^{j \theta_{3}}+\overline{F_{4}} e^{j \theta_{4}}$

The solution of Eq.(A.5) is

$$
u_{0}=\left\{A_{5} e^{j \theta_{5}}+C . C\right\}+\left\{A_{6} e^{j \theta_{6}}+C . C\right\}
$$

Where $C . C$ is the complex conjugate, and $\theta_{5}=k_{5} x-\omega_{5} t$, $\theta_{6}=k_{6} x-\omega_{6} t$. And $\left(k_{5}, \omega_{5}\right)$ and $\left(k_{6}, \omega_{6}\right)$ meet the dispersion relation $\lambda_{1} k^{4}+\lambda_{2} k^{2}-\omega^{2}=0$.

Substituting the solution in Eq.(A.7) into the Eq.(A.6), the right hand side of Eq.(A.6) has three main source terms. The source terms, $\frac{d u_{0}}{d t}, u_{0}^{2} \frac{d u_{0}}{d t}$ and $\Pi$ include the resonant terms, $e^{j \theta_{5}}$ and $e^{j \theta_{6}}$, and other terms, $e^{j 3 \theta_{5}}, e^{j 3 \theta_{6}}, e^{j\left(2 \theta_{5}+\theta_{6}\right)}$, $e^{j\left(2 \theta_{5}-\theta_{6}\right)}, e^{j\left(\theta_{5}+2 \theta_{6}\right)}$ and $e^{j\left(\theta_{5}-2 \theta_{6}\right)}$. In general, difficulty arises when $e^{j 3 \theta_{5}}, e^{j 3 \theta_{6}}, e^{j\left(2 \theta_{5}+\theta_{6}\right)}, e^{j\left(2 \theta_{5}-\theta_{6}\right)}$, $e^{j\left(\theta_{5}+2 \theta_{6}\right)}, e^{j\left(\theta_{5}-2 \theta_{6}\right)}$ happens to be natural mode.

The solution in Eq.(A.5) can be revised with multiple time scale

$u_{0}=\left\{A_{5} e^{j \theta_{5}}+C . C\right\}+\left\{A_{6} e^{j \theta_{6}}+C . C\right\}+\left\{A_{7}(T) e^{j \theta_{7}}+C . C\right\}$

With $T=\epsilon t$. The new time scale $T=\epsilon t$ is determined from the naïve expansion.

Substituting the Eq.(A.8) into the Eq.(A.6), and let the resonant terms in right hand side of the Eq.(A.6) be zero and obtain the solution of $u_{1}$. And $e^{j \theta_{7}}$ may be anyone of $e^{j 3 \theta_{5}}, e^{j 3 \theta_{6}}, e^{j\left(2 \theta_{5}+\theta_{6}\right)}, e^{j\left(2 \theta_{5}-\theta_{6}\right)}, e^{j\left(\theta_{5}+2 \theta_{6}\right)}$ and $e^{j\left(\theta_{5}-2 \theta_{6}\right)}$. Where $\theta_{7}=k_{7} x-\omega_{7} t$ and $\left(k_{7}, \omega_{7}\right)$ also meet the dispersion relation $\lambda_{1} k^{4}+\lambda_{2} k^{2}-\omega^{2}=0$. 